
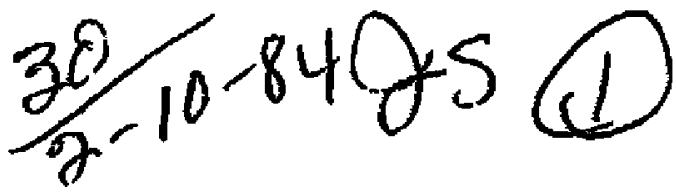

SLAC-Ptb-32A1

January 1984

(T/E)

$$
\text { SCR }
$$

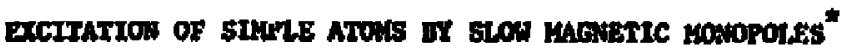

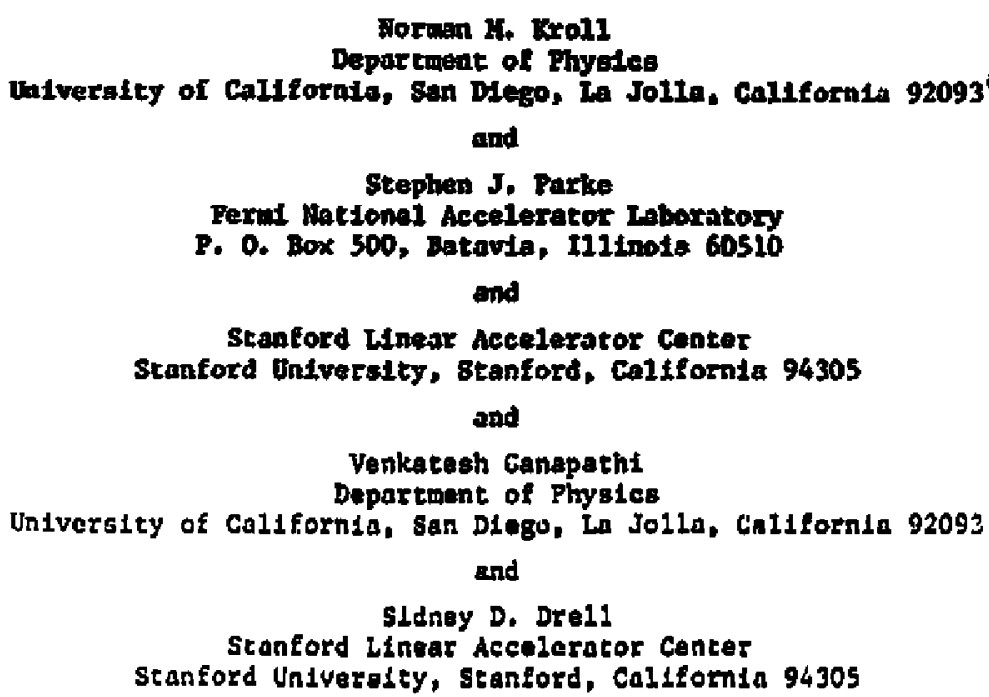

\title{
ABS TMCT
}

We pteacnt a theory of excication of aipple atoma by slow muving massive monopules. Previously presented rasulte for n monopole of Ditac strength on bydrogen and holium are raviewed. Thu hydrogen theory is extended to Include arbitrany integral multiplas of the Ditac pole scrength. The excitation of helium by doubla strangth poles and by dyons ts also discussed. It is concluded that a hallum propoctional counter 1 s a reliable and effective detactor for monopoles of arbltrary atrangth, and for negatively charged dyons.

* Hoxk supported In part by tho baptrtment of Buetgy, contractes DE-AC03-76Sroos15 (STAC) and DE-AT03-81ma0029 (UCSD). Fernilab is operated by the thivergitiea hesearch Aedocintion Inc. under contract with the United States Department of Energy.

Invited vaper presented by H. H. Kroll and S. 3. Parte at the Monopole ' 83 Confexence, Am Arbor, Hichtgan, October 6-9, 1983. 
$x-34 k$

$=1,13 \%$

8310 xi;

I. DNTRODUCTION

As dibcussed by Drell et al, (hereafter referred to an DKMPR), massive Dirac monopoles bave a large effect on atomic energy levels, and can cause degeneracy or near degeneracy between the ground state and excited states of the atom. This phenomepon leads to greatly enhanced excitation cross sections for slow moving monopoles. For simple atoms the effect can be reliably calculated, thve prowiding the poublbility of reliable low $\beta=v / c$ detection. What is believed to be a quito occurnte calculation has been carried out for a minlmum atrength monopolo in hydrogen, and a calculation of uncertain accuracy has bean carrlod out for such a monopole in helium.

In the following we shall revtew the method and reoult of DKMPR, discuss some further inveatigation of the hydrogen ense which includes an extension to monopoles of arbitrary mutiples of the Dirae charge, and describe work in progreas which should lead to comparably accurate predictions for heliurn. We shall also discuss the interactlon of double strength poles and dyons with helium. Finally, we shall conclude with a few comments about the potentlal utility of other noble gas atoms as monopole detectors.

\section{THE BASTC MECHANISM AND COMPUTATIONAL STRATEGY}

In the following we treat the monopole an infinttely massive. and, untll we discuss recoll effects, the meileng at infinttely massive. We vee 
non-relativietic theory and atnume the normul Dirac megnetic moment for the olectroas. The Hamiteonian for on atom may then be written

$$
H=\sum_{i=1}^{x} \frac{\left(p_{1}-A\right)^{2}}{2 m} \cdot \frac{a}{2 m} g_{1} \cdot g_{i}+v\left(x_{1}+\cdots x_{i}\right)
$$

In (2. 1) we have

$$
\begin{aligned}
& \underline{B}_{i}=\frac{g f_{i}}{r_{i}^{2}} \\
& A_{i}=g g_{i} \frac{\left( \pm 1-\cos \theta_{1}\right)}{r_{1} \sin \theta_{1}}
\end{aligned}
$$

The two signe in $(2,3)$ reler to alternate gaugos and $\left(r_{1}, \theta_{1}, f_{1}\right)$ are spinerical coordinates for,the $1^{\text {th }}$ electron relative to the pole, Finally, $U$ repre. eentg the electrotalic interaction botwen the olectrone and between the electrons and nucleus,

The angula- momentum operator for thit syotem may be written ${ }^{3}$

$$
J=\sum_{i}\left(\underline{x}_{i} \times\left(\underline{p}_{i}-t_{A_{i}}\right)+\frac{1}{2} g_{i}-q \hat{r}_{i}\right)
$$

where $q=e g$ mat be an integer or half integer. The term $y_{l} \times\left(\underline{p}_{1}-e A_{i}\right)$

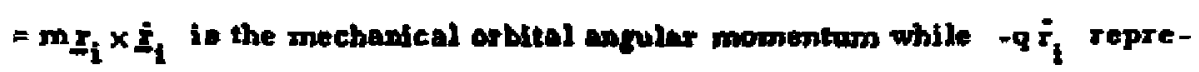
pente the apgular momentum ansociated with the olectrod's electrostatic field crosed with mapnetic fleld of the pols. 4

Becaune of the coordintes we have ueed in the gbove equatione, and also becanse the pole is astrmed to be much more magive than the atom. 
it is conventent to work in a reference frame in which the pole is at rest. If the atom impingee on the manopole with zaro impect perameter oo that the

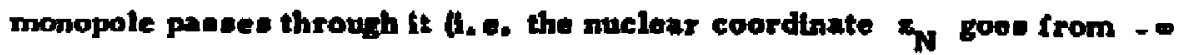
to $+-1 f_{z}$ is conesrved. Slace, however, the $z$ component of field angulax momentum changet from +92 to $-\mathrm{g} 2$ in this procese, the mechanical angular momentum and aptn. which we ldentify with the otomic angular momentum when the atom to out otde the range of the pole's magnetic fleld, must change so as to compensate. Thus $J_{,}$(ator) increased by $2 q z$ at a regult of the collision, Let us suppose that the inclident atom has atomic mpin zero, that is to say the atomile ground state has zero angular momostum (as would be tice case for a noble gat alom). Then no mather how alowly the colligion takes place, after it is over the atom muat bo in a utato of angular momontum greater or equal (typicaly equal) to $|2 \eta z|$, and hesec in an excited state. Even if $J$ (atom) te not zero for the ground state, nome of the magnetic sub. state must beconte exclted. Fo. ple, if the incitont atomic apln is $1 / 2$ then half the atoms $4:$ exeited for $|2 q z|=1$, and all for any Inrger ....te.

To see what ho spens when the impact parameter is nonzcro, we first note that when the pole and nueleus coincide, all three componente of $J$ commute with the Hamitonias and henet the otates may be characteristed as eigenstates of $\mathrm{J} \cdot \mathrm{J}$ with eigenvelut $\sqrt{\mathrm{JJ}+1)}$ and $2 \mathrm{~J}+\mathrm{l}$ degeneracy. Thus the atomic spin zero incident atom finds itself in a atate of degeneracy of at least (typically, prectecly) $|2 q 2|+1$ so it pavaes over the nuclens, In the case of nonzero impact pas ameter it to convenient to ute time dependent coordinate 


\section{DISCLAIMER}

This repont wa prepared as an zscount of work sponsoxed by an Bgency of the United States Government. Neither the Uniled States Gowenment nor bny agency thercof, net any of their employtes, makes any wartanty, enptrats of implted, or noumes any tegal liability or responsibility for the aceuracy, complsteness, or usefulness of any information, apparatus, product, or process disclosed, or represents thet its use would not infringe privately owned rights. Reference herein to any specirle commercial product, Frocess, or iervice by trade name, trademist, manufacturer, or otherwike does twot aecessarily constitute or imply its endorsentat, recommendution, of fovoring by the Unitei States Government of any agency thercof. The views and opinions of authors jexpressed berein do not necessarily state or reflest those of the United States Gojernment or any agency thereof.

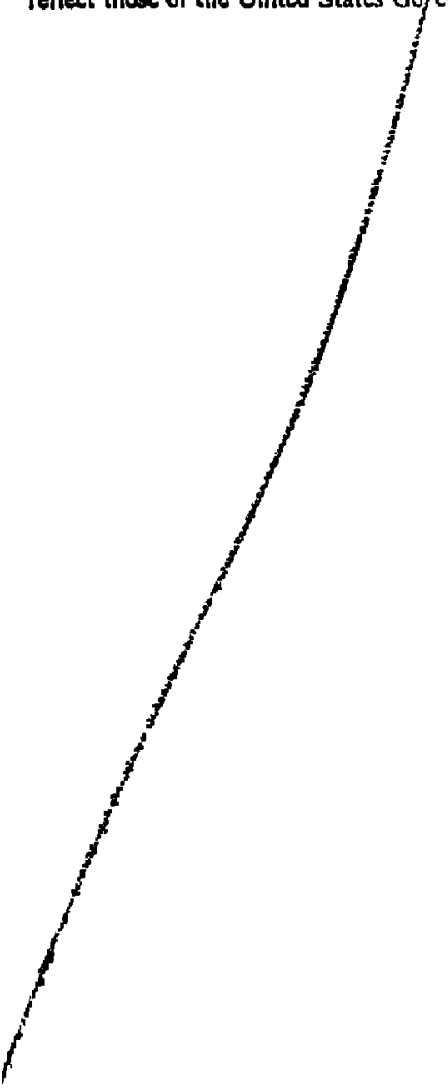


ayotem in which the nucleus is on the negative $z$ axis. In that case the instantaneous states are atill characteriged by the elgenvalues of $J_{z}$. Furthermore, for adiabatic motions transition between states of different $\mathrm{J}_{2}$ will be very improbable unless degeneracy or near degeneracy occura. (Note that constunt $J_{z}$ here means that the atom remaing in its ground atate. This differo from our previous diseussion because here $j_{z}$ refers to a $z$ axds which has a different direction before and after the collision.) The previous discusion, however, telfa us that for gmall impact parameter the spin zero incoming atato will become nearly degenerate with the $|2 q z|$ partmers which it would have of the center. Ouasi-adiabatic transfers to the se states hecome probable and lead to excitation. Additional duencracies Will oceur if the $z_{N}=0$ atate to which the incident ground state connects is not the ground state of the $z_{N}=0$ Hamiltonian. These additional degeneracies, whlch we refer to as off center level crossings, occur for a range of impact parameters, and can provide an additional source of excitation. They may enhance tho excitation cross sectiona for double strength, fes on He. and they are expected to be important for the heavier noble gas atoms. For nonzero incident spln the situation 10 similar but different magnetic substates must be treated separately as they connect with different $z_{N}=0$ states, and the degree of degencracy of the $z_{N}=0$ state varies vith magnetic subotate. Whth the above pleture in mind, a general strategy for calculating excitation cross sections may be described.

(1) The $z_{N}=0$ low lying energy level gystem must be established. This is a relatively simple task for one and two electron atoms. A 
substantial effort would be involved to do it reliably for such thinge as Ne and $A$. Connections between these stated and the otates at $z_{N}= \pm 0$ can be ectablished by assuming that states having the eame eigenvalue of $\mathrm{J}_{\mathrm{z}}$ do not cross. These connections ere Eufficient to establish whether or no: there are off center crossings and to determlne which states will be excited,

(2) Excitation transitions via the central multiplet can bie calculated by means of a simple extension of the Landat-Zener theory. 5 To obtaln quantitative results for a given distance of closest approach, and veloctry at that distance, one requites only a knowledge of the central multiplet aplitting which occurs at distances of that arder. We absume here that these transitions are probable orly if the eplitting is 5 mall, and honce lines in the separation. It can therefore be obteined by applying firet order perturbation' theory to the central multiplet, provided a suciciently accurate form for the wave function of the central multiplet can be obtatned.

(3) Excitation sial of center crossing are aignificant only if $\Delta \mathrm{J}_{\mathrm{z}}= \pm 1$ for the crossings, a circumstance which has, among the cases we have studied, hed occurred only for double strength polus on He. Such crosings are also expected to occur in more complex systems such as Ne and $A$. They are most likely to lead to transitions when the diatance of clogest approach is equal to the crossing distance. Henze the determination of this distance and the wave function is needed. as well as an appropriate treatmest of the differ. ential equation which couples the states,

(4) Since we are treating the orbit of the incoming atom clasuically. we can use the dependence of the energy level on $z_{N}$ to determine an 
effective potentlal between the ptom and the pole. For excitation via the central ronitiplet, one is primarily interested in relating the diatance of clonent epproach and the atomle velocity at that diftance to the velocity and Impect parameter at $z_{N}=-\infty$. Thle can be determined to a good approximation from the informatton already determined in (1) and (2), and together with (1) and (2) provido a practieal prodedure for determining the excitation erosa dectiona. Mors detalled information about the energy as a function

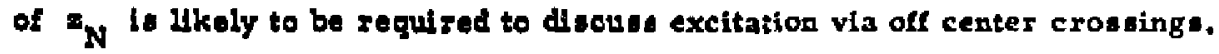
The ganeral procedure desarlbed above will be illustrated and further explatred in the application to be deseribed in subsequent cections. Before dolng Bo, howtever, wo explatn the $\Delta \mathrm{J}_{\mathrm{z}}= \pm 1$ selection rule for off center croolinge. Wo tirat imgine the eigenvalues and wave functione of the Iramflentan whth the nuoleun on the negative $z$ axis to be known. The states are also elgenstates $\mathrm{J}_{2}$, and as mentioned before, states of the ame $\mathrm{J}_{2}$ wll not erose so that a wique energy $\Sigma_{n}\left(\varepsilon_{N}\right)$ can be defined for each state $n$. We next conslder the eave of the nueleut moving in some orblt in the $x=0$ plene, and daline a primed coordinate syetem whose $x^{\prime}=0$ plane colncides Wth the $x=0$ plane (i.e. $\left.x^{\prime}=x\right)$ and whose 2 axis pointe from the pole to the nueleus. The Hamltonlan in these primed coordinated hat the same form as $(2,1)$ with auelenr coordinate $\mathrm{IN}_{N}^{\prime}=\left(0,0,4 \mathrm{E}_{N}\right)$. In the re varlables the Hamiltiofian ha en oxplielt time dependence due to the variation of $z_{\mathrm{N}}$ with time and an implicit thme dependence dive to the time dependence of $y^{\prime}$ and

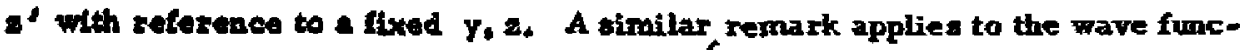

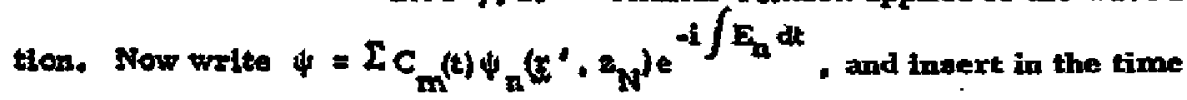


dopendent Schroedinger equation to obtain 6

$$
\frac{d C_{n}}{d i}=-\sum_{m}\left(\psi_{n} \cdot \frac{d \psi_{m}}{d t}\right) e^{-t \int\left(E_{m}-E_{n}\right) d t} C_{m}
$$

The qual adiabatic approximntion coaviete of restrictiog the velues of $\mathrm{n}, \mathrm{m}$ to refer to the pair of levela which are crouging. Whth the natural choice of

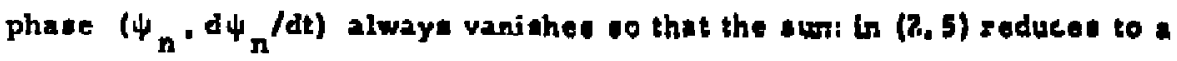
tingle term, and we bave a pair of coupled equatione for $C_{n}$ ond $C_{m}$. Next we obzerve that

$$
\frac{d \Psi_{m}}{d t}=\left(-i \frac{y \cdot \dot{y}^{\prime}}{z_{N}} L_{x}^{\prime}-f^{\prime} \cdot y \frac{d}{d z_{N}}\right) \Psi_{m}
$$

where

$$
L_{x}^{\prime}=\sum_{j}^{z} \underline{r}_{j}^{\prime} \times \underline{p}_{j}^{\prime} \cdot \dot{x}^{\prime}
$$

Now $\left(\psi_{n}, d \psi_{\text {rar }} / \mathrm{dz}_{\mathrm{N}}\right)$ vanishes tolew $\Delta \mathrm{J}_{z}^{\prime}=0$, whlch never hold for croat-

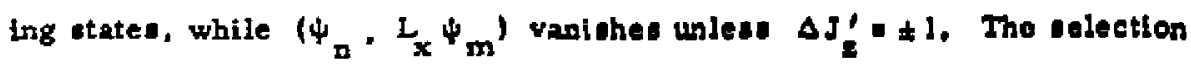
rule for off center crousinge is thus explained.

\section{TIIE HYDROGEN ATOM}

The simpleat illuatration of the preceding dicueualon is provided by the hydrogen atom. For notational simplieity, we aoume the pole strength parameter q to be positive, and, of course, $z=1$.

a) The energy levels at $z_{N}=0$, where the pole and charge colnetdo, Ia given by* $E=R y / n^{* 2}$ where $z^{*}=1+n_{r}+\sqrt{(j+1 / 2)^{2}-q^{2}}, y=q-1 / 2$, $q+1 / 2, \ldots$ and a linearly independent set with $a^{*}=n_{y}+\sqrt{(j+1 / 2)^{2}-q^{2}}$, 
$J=q+1 / 2, q+3 / 2, \ldots$, in both caees, $n_{p}=0,1, \ldots$ is the number of radial nodes. Since for large $J$

$$
\sqrt{(J+1 / 2)^{2}-q^{2}}=J+\frac{1}{2} \cdot \frac{q^{2}}{2 J+1}
$$

and thus $n^{j}$ depende primarily upon $n_{r}+J$, the pattern of $J$ degeneracy and multiplet structure is similar to that of the pole frec case.

The ground state, with $E=-R y, J=q-1 / 2$; and the flrst excited state, with $E=-R y /(2 q+1), J=q+1 / 2$ are the two $z_{N}=0$ states which connect to the ground state at $x_{N}=-\infty$, and correspond to the central multiplets referred to in section $I 1$. The state with $J_{z}$ (atom) $=-1 / 2$ at $z_{N}=-\infty$ has $J_{z}=q-1 / 2$. If the collision is at zero impact parameter, it connects to the lowest state with $J_{\mathrm{z}}$ (atom) $=2 q-1 / 2$ at $z_{\mathrm{N}}=+\infty$. For $q=1 / 2$ this is simply the $+I / 2$ component of the ground state and no axcitation 1s involved. For layger q excitation must occur. For collisions in which the Impact parameter is nonzero but sufficiently small, the $2 q$ cornpoments of the ground state never actually cross but come sufficiently close together to allow quasi adiabatic tranefer to occur among them, leading to a dietxibution of excited final states with $2 q-I / 2 \geq J_{2}$ (atom) $\geq 3 / 2$. The atate with $J_{2}$ (atom) $=+1 / 2$ at $z_{N}=-\infty$ had $J_{2}=q+1 / 2$ and hence must connect to the $J=q+1 / 2$ state, Thus as $z_{N}$ varies from - o to zero, its energy increases from $-R y$ to $-R y /(2 q+1)$. At the oume time there are $2 q-1$ states which descend from $z_{N}=-\infty$ excited states to the $z_{N}=0$ ground atate whth encrgy -Ry and hence experience off center crozsings with the $J_{2}=q+1 / 2$ state, These off center crossings are with states having 
$q-3 / 2 \geq J_{2} \geq-(9-1 / 2)$. Thus a tranter to any of these states involved $\Delta J_{z} \geq 2$, and hence do not occur in the quasi adiabatic regime. Hence we confine our attention to excitation via the cent ral multiplet.

In order to diecusg excitation via the central multiplet, we first

writc the Hamiltonian as

$$
I I=\mathrm{H}_{0}+\mathrm{H}_{\mathrm{c}}
$$

where

$$
\begin{aligned}
& H_{0}=\frac{(P-c A)^{2}}{2 m}-\frac{q}{2 m} \frac{g \cdot \hat{r}}{r^{2}}-\frac{c^{2}}{r} \\
& H_{0}=\frac{-c^{2}}{x^{2}} \hat{r}+I_{N}
\end{aligned}
$$

We are assuming the pole to be fixed at the origin and the distance $r_{N}$ of the proton from the origin to be auficiently small to allow us to repreaent the change in the electrostatic potential by the electric dipole approximation. Furthermore, we wite

$$
\underline{r}_{N}=v_{0} t \hat{z}+b_{0} \hat{x}
$$

where $b_{0}$ is the distance of closest approach and $y_{0}$ ie the velocity of the nucleus when it is at that distance. Thus we are neglecting orbit curvature and velocity variation near the point of closest approach. Degenerate first order perturbation theory applied to the eubapace formed by the central muliplet can be writen

$$
i \frac{d \psi}{d t}=\gamma\left(v_{0} t J_{z}+b_{0} J_{x}\right) \Psi
$$


where

$$
\begin{aligned}
v & =-\frac{e^{2}}{J(J+1)}\left(\frac{t+5}{z^{2}}\right) \\
& \left.=-\frac{e^{2}}{J(J+1)}\left(\frac{1}{2} \leqslant \cdot g-q\right) / x^{2}\right)
\end{aligned}
$$

In $(3,4) \psi$ is a $2 J+1$ component column makrix whose componente are the ampitudes of the $J_{g}$ eigeratates, and $J_{F}$ and $J_{x}$ are angular momentum matrices in the standard $J$ representation.

Sotting

$$
\psi=\left(\begin{array}{c}
c_{J} \\
\dot{c}_{\mathrm{M}} \\
\dot{c}_{-J}
\end{array}\right)
$$

we obtaln

$$
\begin{aligned}
i C_{M} & =\gamma v_{0} t M C_{M}+\frac{\gamma b_{0}}{2}\left(C_{M+1} \sqrt{(J-M)(J+M+1)}+C_{M-1} \sqrt{(J+M)(J-M+1)}\right) \\
& =J \leq M \leq J
\end{aligned}
$$

The Ansate

$$
c_{M}=\left[\frac{(2 J) !}{(J-M) !(J+M) !}\right]^{1 / 2} z^{J+M} \psi^{J-M}
$$

solves these tyublows purtuled

$$
\begin{aligned}
& t=-\frac{1}{2} i \gamma \gamma_{0} t u-\frac{1}{2} I \gamma^{b}{ }_{0} v \\
& v=\frac{1}{2} i \gamma \gamma_{0} t v-\frac{1}{2} i \gamma b_{0} u
\end{aligned}
$$


Equations (3.9) and (3,1D) are equivaleot to Zener's (eq. 1). ${ }^{5}$ we seek a colution far which $C_{I}=1, C_{M}=0(M+J)$ at $t=-\infty$. Thlo 1s abtained by wolving (3.3) and $(3,10)$ with $v(-\infty)=1, \nabla(-\infty)=0$, These are fugt the Zener boundary conditions, so that we can adopt his eolutions. Since we only need the the $t=+\infty$ values, we writs

$$
\begin{aligned}
& \left|u^{2}(+\infty)\right|=\exp \left(-+\cdots b_{0}^{2} f\left(2 v_{0}\right)\right. \\
& \left|v^{2}(+\infty)\right|=1-\left|u^{2}(+-)\right|
\end{aligned}
$$

which yields at $t=t$

$$
\left|C_{M}\right|^{2}=\frac{(2 J) !}{(J-M) !(J+M) !} \exp \frac{-\pi \gamma b_{0}^{2}(J+M)}{2 v_{0}}\left(1-\exp \frac{-\pi \gamma b_{0}^{2}}{2 v_{0}}\right)^{J-M}
$$

It will be shown below that the impect parameter $b=\lambda b_{0}$ and tho Incoming -1, it: $v=v_{0} / \lambda$, where $\lambda$ lo a furetion of $v$ which we shall dotermine. Assuming this to be the case here, we obtain the partial excleation cross sections

$$
\begin{aligned}
\sigma_{M} & =\pi \int d b^{2}\left|C_{M}\right|^{2}=\lambda^{3} \frac{2 v}{Y} \int d x e^{-(J+M) x}\left(1-e^{-x}\right)^{J-M} \frac{(2 J) !}{(J-M) !(J+M) !} \\
& =\frac{2 y \lambda^{3}}{Y(J+M)}, J \geq M \geq-J+1
\end{aligned}
$$

If whould be clear that the above theory of excitation via a cestral multiplet applies to any atom. The quantitative problem in the caee of complex ntoms arises in the evaluation of $Y$ and $\lambda$. For the cace of hydrogen. we find 


$$
Y=4 \alpha /(2 q+1)
$$

for the $\mathrm{J}=\mathrm{q}-1 / 2$ state, and

$$
\gamma=4 q q /\left[(2 q+3)(2 q+1)^{2}\left(2 q+1-\frac{1}{2} \sqrt{2 q+1}\right)\right]
$$

for the $J=q+1 / 2$ state, Here $q$ is the fine structure contant and in our units $\gamma$ is an inver Be length squared, so that the units are inverse Bohr radif squared.

To determine $\lambda$, we assume the motion of the proton may be deacribed classically and that it is deternined by studying motion in the potcntials $V_{-}=E\left(q-1 / 2, \underline{s}_{N}\right)$ and $V_{+}=E\left(q+1 / 2, I_{N}\right)$ where $E\left(q+1 / 2, \underline{J}_{N}\right)$ is the suinimum elgenvalue of the Hamilomian with $I_{N}$ held flxed and $J_{z}$ with eigesvalue $q \neq 1 / 2$. Here $J_{z}$ refers to the componen. of angulat momentuni along an axis directed from the pole to the proton. Since the potential depends only on $\left|y_{N}\right|$, angular momentum is conserved in the motion, yielding $v b=v_{0} b_{0}$. The ratio of $v$ to $v_{0}$ is given by energy conservation. In order to obtain $a b$ independent form for $x$, we have asoumed $v_{ \pm}\left(b_{0}\right)=v_{ \pm}(0)$. With these ac sumptons, $\lambda=1$ for the lower state and

$$
\lambda=\left(1-\frac{2 q q^{2}}{(2 q+1) v^{2}}\right)^{1 / 2}
$$

for the upper state. Evidently $\lambda$ depends culy upon the excitation energy of the central multiplet state so that its evaluation for complex atoms depends only upon a determination of the excitation energies. 
The total excitation cross section to thus given

$$
\begin{gathered}
\sigma=\frac{1}{2}\left(\sigma_{-1 / 2}+\sigma_{1 / 2}\right) \\
\sigma_{-1 / 2}=(q+1 / 2) \frac{\beta}{\alpha}\left(1+\frac{1}{2}+\cdots+\frac{1}{2 q-1}\right) a_{0}^{2} q>1 / 2 \quad(3,18) \\
\sigma_{! / 2}=\frac{\beta}{2 \alpha q}\left(1-\frac{2 q \alpha^{2}}{(2 q+1) \beta^{2}}\right)^{3 / 2}(2 q+3)(2 q+1)^{2}\left(2 q+1-\frac{1}{2} \sqrt{2 q+1}\right)\left(1+\frac{1}{2}+\cdots+\frac{1}{2 q+1}\right) a_{0}^{2}
\end{gathered}
$$

These last expressions have been written in otandira units is that $v$ has betn replaced by $\theta=\mathrm{v} / \mathrm{c}$ and the Bohr radius factors have been put in explicitly. The factors $(1+1 / 2+\ldots)$ come from the sum over partial eross-sections, and in the form witten it is assumed that all are above threshold, For example, for $q=I, \sigma_{-1 / 2}$ has only one term and represent s excitation to the $n=2$ level with $(v / c)=(a / 2) \sqrt{3 m_{e} / m_{p}}$ at threahold. $\sigma_{1 / 2}$ has three terms, representing excitation to an $n=2$ level and two excitations to $n=3$ levels with a cli ghtly higher threshold, For fixed $n, \lambda$ determines the threshold when the energy of the relevant central multiplet exceeds that of the final atate, that is when $2 q+1>n^{2}$. The excltation erose sections increase quite rapidly with $q$, and our approximatione lose thelr valldity al crose sections become of order $a_{0}^{2}$. As was emphasized in DKMPR, the cross section is quite large even for $q=1 / 2$. 


\section{THE HELTUM ATOM}

The oimplent syatem of practical Interest to which our theory applies is the helium atorn. We obtain reaults in this sectlon for the $q=1 / 2$ and $q=1$ case.

(a) The $q=1 / 2$ case

The lowest two hydrogeric states fcr $z_{N}=0$ have $j=0$ and 1 . Thus in the shell model approximation the ground state will be an antisymmetrized product of $j=0$ and $J$ orbitals yiclding a ground atate of $\mathrm{J}=1$. This state, which provides the central multiplet through which transitions occur, has a binding enexgy which we have variationally estimated to be $8.49 \mathrm{eV}$, so that the He atom sees a repulsive barrier of height $16,09 \mathrm{eV}$. This leads to a threshold factor $\lambda=\left(1-\beta_{c}^{2} / \beta^{2}\right)^{1 / 2}$ with $\beta_{c}=9.29 \times 10^{-5}$. The excitations are to the lowest ${ }^{3} S_{1}$ and ${ }^{3} P_{2}$ states. These occur in the ratio $2: 1$ with thresholds at $\beta \times 10^{4}=1,03$ and 1.06 , respectively. Applyiser eq. (3.14), the excitation cross section is given by $\sigma=\frac{2 v \lambda^{3}}{\gamma}(1+1 / 2)$. The critical problem remaining is the determination of $\gamma$.

A preliminary determination has been made using a product wave function whose spin and angle dependence is the same as that of the hydrogenic orbitals and whose radial parts take the form

$$
\begin{gathered}
f_{0}=e^{-a_{0} r / a_{0}} \\
f_{1}=x^{\sqrt{2}-1} e^{-a_{1} r / a_{0} \sqrt{2}}\left(1-t \frac{x}{a_{0}} e^{-w r / a} 0\right) \\
-I 5-
\end{gathered}
$$


In these expressions, $a_{0} * a_{1}, t$, and $w$ are parameters determined by minimixing the energy subject to the constraint ${ }^{9}$ that the electric and magnetic dipole forms of the perturbation energy give the same result. While they are guaranted to give the same realt if the perturbation ia computed using exact wave functions of the Hamilonian, they are in gereral not the ame for approximate wave functions. The value obtained for $\gamma 184.66 \gamma_{\mathrm{H}}$ where $\gamma_{H}=a / 4(4-\sqrt{2}) a_{0}^{2}$. The result reported in DKMIR, 2. $35 \gamma_{H^{\prime}}$ was obtained from the magnetic dipole form, uring a crtder wave function, namely (4.2) with $t$ set equal to zero. The cruder wave function with the electric dipole form yields $3.53 \gamma_{\mathrm{H}}$. While we believe the new value to be the more reliable, the results above have convinced us that a more elaborate and systematic approach is desirable. The computational problem is quite similar to that involved in computing the fine and hyperfine (He ${ }_{3}$ ) atructure of the helium $P$ states, which has been done to great accuracy, and we are in the proceas of adopting the methods used there ${ }^{10}$ to this problem. It Bhould be possible to assess the accuracy of a given level of approximation for the wave function by comparison with the helium hyperfine structure results and by comparing its results for the electric and magnetic dipole forms of the interaction. For the present, hotwever, we consider our be at value to be

$$
\sigma=2.5 \times 10^{-18}\left(8 / 10^{-4}\right)\left(1-\beta_{c}^{2} / \theta^{2}\right)^{3 / 2} \mathrm{~cm}^{2}
$$

with $\beta_{c}=9.29 \times 10^{-5}$. 
(b) The $q=1$ ears

The loweat hydrogrifle thee for $z_{N}=0$ hive $j=1 / 2$ and $3 / 2$. Thus the shell model ground otate is obtained by putting both electrons in the ground state, yielding $\mathrm{J}=0$ growd atate. This atate connects with the

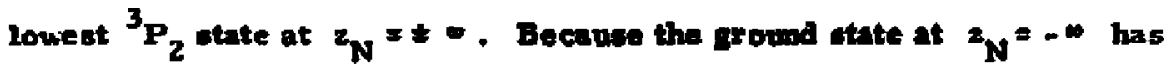
$I_{2}=2$, the central multiplet which ff convecta is the $I=2$ level formed by the (1/2)(3/2) configuration. Thit confguratlon also he $t=1$ level and it is important to know which of the two lies lower. A stmple vertational calculation seems to provide convinctus ovidence that the $J=1$ ievel it lower by about $0.9 \mathrm{eV}$. There are therefore fow off center croosbe by the $J_{z}=2$ state as $z_{N}$ varies from - to zero. The first, which occurs at $z_{N} \approx a_{0^{r}}$ is with the $J_{z}=0$ state which connecte the ${ }^{3} P_{2}$ etate at $z_{N}=-\infty$ to the $J=0$ ground state at $z_{n}=0$. Thare is no quel adibbatic trangfer at this c5osing because $\Delta J_{2}=2$. The other three crosalinge are with the $J_{2}=1,0,-1$ compenents of the $J=1$ central multiplet. The $J_{2}=1$ erousing, which gatiefies the $\Delta J_{z}= \pm 1$ rule, occure at $z_{N} \sim 0.10_{0}$ and 10 a petantlal additional source of excitation. More expelul examination of the opectrun at omall $z_{N}{ }^{12}{ }_{N}=$ $0.5 \mathrm{a}_{0}$ and legs) reveals a mote complex etuntion than wo have diccussed previously. Tho coupling of the electric alpole perturbation to the tuner electron is so strong that it brenks the quartat-daublat couplias for ${ }^{4} N^{20.003} a_{0}$. Since such distancer make a nagligible contribution to the crose section, one can confine one's attention to the "Pashon-Buch" region in which the inner electron hae $j_{10}=1 / 2$ and the outer electron he $j_{2 n}=3 / 2$. The levelo 
which concribuce to the exelcation proces are jutt tha $\rfloor_{4},-3 / 2,-1 / 2$, $1 / 2$ levels of the outer electron. The electroscacle interaction berreen the innex and outer electrons dons, however, reduce the spactog betreen levels for $z_{y} \geq 0.080_{0}$, with the rovereal of level ordet tateng place at $z_{\mathrm{Y}} \approx 0.08 \mathrm{a}^{*}$. We have not worked out the detals of the excitation process for this more cowplex struation, but it 10 clear that apptication of the central woliplet theors to the encttod electron will yield a lorer 1init for the cross sections, comparing $x$ valuag for $q=1 / 2$ and $q-1$ and using (2. 16). wo aue that the $q=1$ monopole is at ladbe a lector 2.3 more effective than the $q$ - $1 / 2$ monopole. Thle gector will be anhancud not only by che electrostatic mareoring of the lovel goparations but al wo by the fact that the screening wi1l be atronger and tha fact thut ancra is an additional level. The excitatcons are to ${ }^{3} s_{1}, 3_{2}$, and $3_{3}$ wth $\times 10^{\text {th }}$ thresholds at 1.03 , 1.11. and :.11, respectively. 11 Whilo ona might 1magine that the threshold factor $\lambda^{3}$ mighe be santier at threahold dut to the race that the central

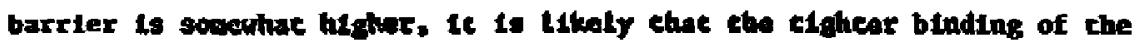

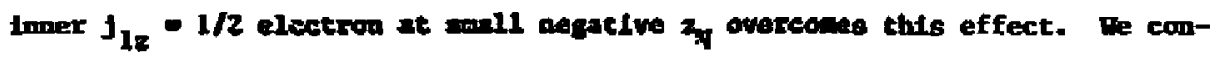
clude, therefore, tha' Ee is a vay affective datector for $q$ - 1 mopole and

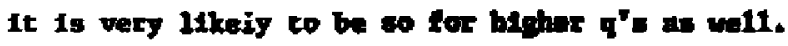


As discussed in ref, 1 and by several epeakers at this conference, the preferred method of detecting the excitation is by colliaional ionization of the ${ }^{3} \mathrm{~S}_{1}$ states with a doping gas ouch as $\mathrm{CO}_{2}$ or $\mathrm{CH}_{4^{*}}$ All of the bigher triplet states quickly decay elther directly or by cascade to the metastable ${ }^{3} s_{1}$ state.

V. DYON INTEKACTIONS WITH HELIUM

In this section we consider the interaction of a dyon with helium. The fron is as bumed to have $q=1 / 2$ and plus or minus one unit of eleatric charge. Such a charge on a monesole could arise during the production mechanism in the early universe or in the case of positive charge by the subscquent capture of a proton. The size of the monopole -proton bound state is approximately $10 \mathrm{fm}$ and is therefore very small compared to atomic dimensions and hence will be considered point like,

The positively and negatively charged dyons need to be considersd separately, and we first consider the positive charged case. Following the discussion of sections $I I$ and $\amalg$, the eigenstates of the ryon-helium atom system are first identified at large separation. Apart from the usual states of the helium atom, an electron can form bound states with the positively drarged dyon with energies $-\frac{1}{2} m^{2} /(n+\mu)^{2}$ where $n=0,1, \ldots$ and $\mu=\sqrt{j(j+1)} ; j=0,1,2, \ldots$ being the angular morentum. The ground state of the dyon is $13.6 \mathrm{eV}$ below the continum and thus the dyon will pick up an electron while travelling through matter. Metal surrounding or making up a detector would be an ideal source of such electrons. Therefore we need to 
consider a dyonis atom and a helium atom colliding, i. e. a three electron system.

Next, we consider the dyon and helium nucleve on top of one another. In doing this, we mut ignore for the time being the Coulomb repulsion between the dyon and the nucleur. This interaction, which turna out to be quite important, will be taken into account later.

The relevant $x_{N}=0$ central multiplet of the three electron system is in a $(0)(1)^{2}$ configuration rormed from the two lowest hydrogenic atates and because of the Pauli principle, must have $J=1$. Because one of the electrons is already bound to the dyon (with $J=0$ ) when the colliaion begins, the $J_{z}$ value which connects to the $z_{N}=-\infty$ configuration is $J_{z}=1$. This shows thet the ground state $z_{N}=0$ central multiplet described above is indeed the relevant central multiplet and there are no off center crosings. ihe states which conneci to tne $J_{2}=1$ and $J_{2}=0$ states of the central multiplet when $z_{N} \rightarrow+\infty$ are, as discussed in section 11 , the minimum energy $J_{z}=1$ and $J_{z}=0$ states with $z_{N}=+\infty$. Coe eees by inspection that these are states with He in its ground atate and with one electron bound to the dyon and in excited $J=2$ or $J=1$ states with excitation energies of 11.3 and 6.8 visits, respectively.

Following the method of section III, we may calculate the transition probabilities as a function of diatance of closest approach $b_{0}$ (eq. 3.13). Again the interaction parameter $Y$ (call it ${ }_{D}$ here) mut be calculated. Following the previous calculation for heiium, this is performed variationally 
using the vame wave functions as in section IV but with the extra electron in a $j=1, m_{j}=0$ monopole harmonic. The parameters of the variational wave functions are determined so as to minimize the energy subject to the constraint that the ulectric and magnetic dipoles as discussed earlier give the samte result. The irreraction paranuter ${ }_{D}$ is found to be approximately 20 limes the hyc.rogen value due mainly to the extra charge (partially shielded) at the origin, which comes in as the third power. Thus we find $Y_{D}=1.4 \times 10^{-2} / a_{0}^{2}$.

In order to compute the partial cross gections we use eqs. (3.11), (3. 12), and (3.13). For this case, we obtain

$$
\begin{aligned}
& \left|c_{0}\right|^{2}=2\left(e^{-\Gamma}-e^{-2 \Gamma}\right) \\
& \left|c_{1}\right|^{2}=e^{-2 \Gamma}
\end{aligned}
$$

where

$$
\Gamma=\pi y_{D} b_{0}^{2} / 2 \beta_{0}=\pi y_{D} b_{0}^{3} / 2 b \beta
$$

We have used angular momentum conservation of the nucleas orbit in the list equality. In order to compute a cross section, we need to express $b_{0}$ in tern:s of $b$ and $B$. This can easily be done taking account of the coulomt repultion betwcen the dyon and the nucleus, the attractive force of the elec trone, and angular momentum conservation. Thus we have

$$
\frac{1}{2} M v^{2}=\frac{1}{2} M \frac{b^{2} v^{2}}{b_{0}^{2}}+\frac{2 t^{2}}{b_{0}}-\Delta
$$


where $\Delta$ is the get increase in binding energy of the electrons whun the dyon drid nucleus are separated by distances amall compared to a ${ }_{0}$. Here $b_{0}$ islud $b$ are in units of $a_{0}$ and $\Delta \approx 180 \mathrm{eV}$. The cross sections may be valuated by computing

$$
\sigma_{M}=2 \pi \int\left|c_{M}^{2}\left(b_{0}\right)\right| b d b
$$

numerically, where $b_{0}: s:$ mputed from $(5,1)$. It is convenient to express the result as

$$
\begin{aligned}
& \sigma(6,8)=\left(\varepsilon / 10^{-4}\right) n_{1} 2.0 \times 10^{-19} \mathrm{~cm}^{2} \\
& \sigma(11.5)=\left(\hat{F} / 10^{-4}\right) n_{2} 1.0 \times 10^{-19} \mathrm{~cm}^{2}
\end{aligned}
$$

Here $0(6,8)$ represents the excitation cross acction for the $\mathrm{J}=1$ excited state of the dyon, which has an excitation energy of $63 \mathrm{eV}$. It correspords to $J=1, M=0$ in (3.13). SimilarIy, o(11.5) corresponda to $J=1, M=1$ in (3. 13) and represents excitation of the dyon to its $J=2$ atate with excitation energy $11.5 \mathrm{eV}$. The parameters $A_{1}$ and $A_{2}$ reprenent threshold factors, apalogous to the $\lambda^{3}$ whicb appears is $(3,14)$, and are determined by the numerical integratica described above. RApresentative values dre: 


$\begin{array}{cccc}1110^{-4} & \Lambda_{1} & \frac{1110^{-4}}{1} & \Lambda_{2} \\ 2 & 1.4 \times 10^{-3} & 3 & 7.1 \times 10^{-4} \\ 3 & 8.5 \times 10^{-2} & 4 & 4.4 \times 10^{-2} \\ 4 & .48 & 5 & .236 \\ 5 & .86 & 6 & .48 \\ 6 & 1.02 & 8 & .77 \\ 10 & 1.04 & 10 & .88 \\ 1 & 1.00 & 20 & .99 \\ & & 1 & 1.00\end{array}$

Tho dicterence in the behavior of $\Lambda_{1}$ and $\Lambda_{2}$ arises from the fact that $\left|c_{1}^{2}\right|$ pesks at $b_{0}=0$ where the Coulomb suppression is complete, while $\left|c_{0}^{2}\right|$ vantshes at $b_{0}$ and peaks at a firite value. The fact that $\Lambda_{1}$ slightly exceeds wilty in a coseoquence of the fact that the force becomes attractive at large Alutancos.

The exeitation may be detected via the $6.8 \mathrm{eV}$ and $4,5 \mathrm{eV}$ photon $\mathrm{s}$ whlch would be exnltted by these states. The amall cross section, the strong Coulomb ouppresslon tor $3<4 \times 10^{-4}$, and the requirement that one detect photens rather than 1enization makea He rather mattra:tive as a detector of pooltively charged dyons.

For a negativoly charged dyon the eigenstates for a widely scparated dyon and atom are juat the aigenatates of the atom. When the dyon and helium stom are on top of ons arther we are trying to bind two electrons to a charge 
one center with a monopole. This slkuation has similarities to the cases of a hydrogen miaue ion which has one bound state with anergy $-0,75$ o $V$ and a helfum minut ion which does not exist as a bound stafo, Lat uB compare the dyon of charge one and the hydrogen minus lon. The fret olectron goes into a state with mergy $-13.6 \mathrm{eV}$ in both case $\mathrm{g}$, whereas the second electron los the dyon case goes irto a triplet state with energy $-6, B$ \& $V$ (ignoring ohleluing) and into the $\mathrm{n}=1$ state with energy $-13,6 \mathrm{eV}$ (ignoring shielding) for hydrogon jon. Also the shieiding for the dyon case will be larger than for the hydrogen ion so that the mon opole will be bound less than $0.75 \mathrm{oV}$ and the re 10 probably no bound state. The details here are not important as a dyon at a veloctty of $10^{-4}$ c can cause transitions of a few tenths of an clectron volt. Thus the cross section for jonization of helium is juat $\pi$ Umes the Gquare of the distance at which binding becomes a faw tentl.s of an electron valt. This distance is estimated to be of order $1 / 3$ a 0 , giving arosa acetion of $3 \times 10^{-17} \mathrm{~cm}^{2}$. Of course, the Coulomb attraction between the dyon and the nucleus will enhance this effect by drawing the bucleus towards the dyon, The added energy to the electrons due to the seduction in charge near the center ( $<55 \mathrm{eV}$ ) is less than the Coulomb attraction between dyon and nucleus at a distance of $a_{p}$ and is therefore negllglble. The energy loss, neglecting the attraction between dyou and nucleus will be of order $100 \mathrm{MeV} \mathrm{em}^{2} \mathrm{~g}^{-1}$. This is about 50 time minimum ionizing and thes an uncertalnty is our estimate of the crose cectior by a lactor of 4 or more is not important. Also there is no need for a quenchirg gas like $\mathrm{CO}_{2}$ or $\mathrm{CH}_{4}$ for this cate as the dyon jonizts the helivm atom direetly. The threshold for this procese is 
$1,2 \times 10^{-4} \mathrm{c}$ and there lo no reduetion in crose section neat threahold.

Below throchold, the foniation cross section probably continue to be high but accompanied by cepture of the a pasticle and electron by the dyon. This procese ultimately leads to a tightly bound a particle dyon syotem which behaves like a positive dyon.

In ournmary, helium would be a very efficient detector for negatively cherged dyons down to a $\beta$ of $1.2 \times 10^{-4}$, but appears to be much less promising as a detector for positively charged dyons.

\section{Vi, HEA VIER NOBLE GAS ATOMS}

The primary limitation of He as a slow monopole detr ctor is its throshold at $B=1.03 \times 10^{-4}$. One would prefer to be sensitive at least to $0.37 \times 10^{-4}$, the velocity of cocape from the earth. Because our arguments relled heavily upon the spherical symmetry which is obtained when the monopole and nucleus coinclde, it to natura: to consider heavier noble gas atoms. Such atoms are extenoively used in proportional counters and are not subject to the annoying leakiness of He proportional counters. Assuming the threshold to be determined by the excitation energy, which we take to be of the ordur of the lonization energy, one fincs $\left(\beta_{t}=\right.$ threshold $\left.B\right)$
Ne
$\underline{\mathbf{K}} \underline{\underline{\boldsymbol{T}}}$
$\underline{X e}$
$10^{4} \mathrm{~s}_{\mathrm{t}}=$
0,48
0.29
0.19
0.14

It is not, however, clear that we have used a proper measure of the threshold. We recall that the motopole presente a $16 \mathrm{eV}$ central barrier to 
the impinging He atom, a fact which gives rise to the $\lambda^{3}$ threahold factor which appeare in eq. (3.14). This berrier provides a threshold factor, rather than the principal determinant of the threnhold, because it is lower than the required excitation eaergy. We have already noted that the existence of a Coulomb barrier in the He-positive dyon problem more than doubles the effective threshold.

In order to asgess the barxier height queation in a preliminary way, we first consider the diamagnetic repalsion which the atom experiences when it is far from the monopole. Uaing experimental value for the diamagetle susceptibility, we find for $a_{0} / 2_{N} \ll 1$,

$$
E \text { (diamagnetic) }=3\left(a_{0} / z_{N}\right)^{4}
$$

with

$\begin{array}{cccccc} & \text { He } & \text { Ne } & \Delta & \underline{K r} & \text { Xe } \\ \text { HL (ev) } & 1.3 & 4.8 & 130 & 265 & 423\end{array}$

While (6. 1) obviously gives a grnes overegtimate as $z_{N} \rightarrow 0$, it should be noted (see fig. 1 of DKMPR) that for He at $z_{N}=a_{0}$ it is roughly a factor ten too emall. While it would be umwarranted to ansume ten is a univer sal factor, it does seem very likely that the actual barrier at $z_{N}=a_{0}$ is several times the value given by $(6,1)$. We turther comment that an argot atom with $\beta=1.03 \times 10^{-4}$, the He threshold, canot penetrate beyond a $200 \mathrm{eV}$ barrier height, and on the beaic of the above discussion, eeme unlikely to achieve $z z_{N}$ as small as $a_{0}$. It may therefore be out of the 
range at which quasi adiabatic excitation takes place. If this is the care, the quasi adiabatic threshold for argon would be higher than that of He.

Once is sufficiently large to a how barrier penetration, it is certein that degeneracies and near degeneracies will be encountered, and hence quasi adiabatic excitation will take place. The question of the magnitude of $Y$ factors, which depend primarily on the extent to which the last shell electrons are screened for the state which connects to the ground state, is then of crucial importance in determining quantitative cross sections. A contribution from off center crosings may also prove to be important, and distortions of the multiplet structure of the sort found in the $q=1$ case for He may further complicate matte:s.

In summary then, the probability that the threshold for qua si adiabatic excitation can be pushed below $\beta=10^{-4}$ by using heavy noble gas atoms seems to be low. More work is required both to determine what the threshold is and how large the cross sections are. It seems likely, however, that they become satisfactorily large somewhere between $\beta=10^{-4}$ and $\theta=10^{-3}$.

\section{CONCLUIING REMARKS}

Desplte some quantitative uncertainties about cross ection magnitudes, it seems certain that He proportional counters will satisfactorily detect masEive Dirac monopoles of srbitrary charge in the $\beta=10^{-4}-10^{-3}$ velocity range. The threshold increases slowly with magnetic charge but wlll remain below the ionization limit at $\beta=1.2 \times 10^{-4}$. Such counters will also detect negatively charged dyons at this threahold. 


\section{A PFENDIX}

Because our use of the magnetic dipole perturbation has received some criticism, ${ }^{12}$ we add some technical comments about the hydrogen energy Ievel problem here.

Considering the energy as a function of $z_{N}$, the term linear in $z_{N}$ near $z_{N}=0$ can be obtained from either the point electric or point magnetic dipole. Here we restrict the perturbation Hamiltonlan to terms linear in ${ }^{2} N$, which means the $\delta A^{2}$ t.rm is omitted in the magnetic dipole case. As explained in footnote 9. the two forms of the Hamiltodian give the same result.

One mfgth also consider carrying out first order perturiotion theory Wth real dipoles, that 15 two point charges (electric or magnetic) of opposfte sign separated by a distance $z_{N^{*}}$ In this case, our argument for the equivalence of the two cases no longer applles. First order perturbation theory for the real electric dipole can be readily carried out, and one finds a fractional correction to our polnt dipole result of order $z_{N} / a_{0^{*}}$ Higher order perturbation theory would, of course, be expected to yfeld terms of similar order. First order perturbation theory for the real magnetic dipole case is, however, singular. To be speciftc, we consider the case in which the dipole is aligned along the $z$ axis, with the original magnetic pole displaced from the or gin in the positive direction. Then for the $J_{z}=1$ state, first order perzurbation theory can be carried our, and a fractional correction to the point dipole result of order $\left(2_{N} / a_{0}\right)^{2 \sqrt{2}-2}$ is found. Ilowever, for the $\mathrm{J}_{2}=0$ and $\mathrm{J}_{2}=-1$ cases, the 
perturbation integrale diverge. If one fir st performs the radial integrals, which do converge, but carriea out the amall $z_{N}$ expansior before completing the anguiar integration, one find that the linear terms agree with the point dipole result, but the angular integral for the order $\left(2_{N} / a_{0}\right)^{2-\sqrt{2}-2}$ correction diverges. If the poles are displaced to the negative side. it is the $J_{z}=-1$ state rather than the $J_{z}=1$ state which is well behaved. (Note thet the Alfficulty does not depend upon the chofee of ftring direction, which only affecte the dependence of the states, The formal origin of the diffieulty lies in the otructure of the differential equation and has been dlocused in the context of variational esleulations by Tiktopoulos. ${ }^{6} T_{0}$ explain the origln physically we thrst secall that the ordinary spherical hermonlc: vanioh nlong the 2 axie for $m \neq 0$. This vanishing can be underctood ac roulting from the effoct of the repulsive centrifugal potential adeociated with the $m^{2} / x^{2} \sin ^{2} \theta \operatorname{term}$ in the Hamiltonian. In the case of monopole harmonle6, the magnitude of the centrifugal potential changes as one swltches from one sign of the 2 axis to the other, and for the $q=1 / 2$ cese, may vanlah on one side or the other for $\left|J_{z}\right|=1$ or 0 . Accordingly, the wave functions do rot venish for such tates along the entire $z$ axis. Shlfting the position of the poie without changing the states, which is what one does when one cerried out IIr ot order perturbation theory, can shift the repulalve and ainguler centrifugal potential into a region where the wave funation does not vanloh, lesding to the divergent expectation valies described bove. Ihus an attempt to spply first order perturbation theory to a real magnetic dipole voing numerical integration techniques ${ }^{12}$ is bound to lead to 
unreliable and confusing reatits.

As a final remark, it may be of intecent to mention that we hove carried out cumerical varintional calculations for the $J_{z}= \pm 1$ exelted states and the $J_{z}=0$ grand state an the way from $z_{N}=-\infty$ to ${ }^{2} N=+\infty$ using wave functions which guarantee consistency with perturbatios theory at the $\left|\mathrm{z}_{\mathrm{w}}\right|=0$ and infinity limits. (Ouy wave function for the ground atate is the same as that used by Tiktopoulos ${ }^{6}$ and our featle egroe with hit.) These have been done accurately enough to allow us to numerically confirm the value of the alope at the origin to 0,2 pereent. The calculations are useful because they indicate that the departure from linesrity lo emall, linearity being valid within -5 percent out to a ditsnet 0.5 to. This result supports the approximations we have ued in earculating oxcltation probabilities. Amalogous calculations beve aluo been carrlod out for $q=1$. 
Footnote:

2. S, Droll, N, Krol, M, Mveller, S. Parke, and M. Ruderman, Phys, Rev, Lett. 50, 644 (1983).

2. T. T. Wu and C. N. Yang, Nuclear Phys. B107, 365 (1976).

3. M. Flerz, Helv, Phys. Aeta 17, 27 (1944).

4. For divergence free magnetontatic fields and vector potentiala, this field engular momenturn is given by $\mathrm{e} A$, so that the canonical angular momentum $\underline{I}_{1} \times \mathrm{P}_{\mathrm{I}}$ represents the sum.

5. C. Zener, Proc. Roy. Soc. AI37, 696 (1932).

6. G. Tiktopoulos, Phys. Lett. 125B, 136 (1983). Compare with oq. (15) of the above paper. We are including our time dependent cholce of gauge in the definition of our time dependent Hamiltonian and hence do not have the $d n / d x_{0}$ term which appears there. While we think that such a term thould not be included in our treatment, to be on the aafe dide we have verified that it also entiaftor the selection rule.

7. We are uring the phrase "quasi adiabatic" to refer to a situation in which relative velocltiea are slow enough to allow us to assume that exeltations are dominated by traneitions to levels whose energy separation from the incldent state is small compnired to the minimum excitation energy which would be required for the isolated atom. The transitions are actually non -adiabatic. 
